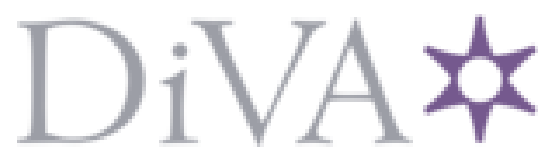

http://www.diva-portal.org

This is the published version of a paper published in Physical Review A. Atomic, Molecular, and Optical Physics.

Citation for the original published paper (version of record):

Jääskeläinen, M., Stenholm, S. (2003)

Localization in splitting of matter waves.

Physical Review A. Atomic, Molecular, and Optical Physics, 68(3): 033607

http://dx.doi.org/10.1103/PhysRevA.68.033607

Access to the published version may require subscription.

N.B. When citing this work, cite the original published paper.

Permanent link to this version:

http://urn.kb.se/resolve?urn=urn:nbn:se:du-11213 


\title{
Localization in splitting of matter waves
}

\author{
Markku Jääskeläinen* and Stig Stenholm \\ Royal Institute of Technology, Roslagstullsbacken 21, SE-10691 Stockholm, Sweden
}

(Received 20 March 2003; published 19 September 2003)

\begin{abstract}
In this paper we present an analysis of how matter waves, guided as propagating modes in potential structures, are split under adiabatic conditions. The description is formulated in terms of localized states obtained through a unitary transformation acting on the mode functions. The mathematical framework results in coupled propagation equations that are decoupled in the asymptotic regions as well before as after the split. The resulting states have the advantage of describing propagation in situations, for instance matter-wave interferometers, where local perturbations make the transverse modes of the guiding potential unsuitable as a basis. The different regimes of validity of adiabatic propagation schemes based on localized versus delocalized basis states are also outlined. Nontrivial dynamics for superposition states propagating through split potential structures is investigated through numerical simulations. For superposition states the influence of longitudinal wave-packet extension on the localization is investigated and shown to be accurately described in quantitative terms using the adiabatic formulations presented here.
\end{abstract}

DOI: 10.1103/PhysRevA.68.033607 PACS number(s): 03.75.Be, 03.65.Ge, 05.60.Gg, 03.65.Nk

\section{INTRODUCTION}

During the past few years, manipulation of confined matter waves has experienced a remarkable progress, both due to the development of laser cooling and trapping of neutral atoms $[1,2]$ and progress in lithographical fabrication of nanostructures for ballistic electrons [3]. In the channeling regime of these experiments, the dynamics will be propagation of matter waves in, possibly multiple, tightly confined wave guides.

Several techniques have been utilized [4] for guiding neutral atoms, among them magnetic confinement both inside hollow glass tubes [5] and above current carrying wires [6], permanent micromagnets [7], light force trapping [8], with microfabricated optics [9] and various other schemes based on the interaction of electromagnetic radiation with atoms. In Ref. [10] the splitting of a thermal atomic cloud above current carrying wires of millimeter size was demonstrated and in Ref. [11] a similar experiment was performed using microfabricated conductors. The creation of Bose-Einstein condensates (BECs) in microfabricated structures was reported almost simultaneously by two groups $[12,13]$ and has since been reported by several groups $[14,15]$. In addition, coherent transfer of a BEC into waveguide structures has been accomplished [16,17]. In Ref. [18] the splitting and propagation of a BEC in a microfabricated optical trap with interferometer structures was demonstrated. This experimental progress has created an interest in the phenomenology of localized quantum states propagating through various types of potential structures.

Interferometer experiments depend directly on the wave nature of quantum mechanics and in such configurations are dominated by the nonclassical properties of material particles. The metrological advantage of using matter waves comes mainly from the increased sensitivity offered by the very short de Broglie wavelengths achievable using laser

*Electronic address: mrq@kth.se cooled atoms [19,20]. Several different setups for atom optical analog of well-known interferometric devices have been proposed and investigated in previous theoretical studies. In Ref. [21,22], waveguide interferometers based on magnetic trapping and a time-dependent bias field were described. The use of time-dependent currents to change the potential configuration was considered in Ref. [23]. The physics of an atomic interferometer in the multimode regime was investigated in Ref. [24]. Other works have investigated the nonlinearities due to many-body effects in atom optics, see, e.g., Ref. [25] and references therein. In Ref. [26] the behavior of a Tonks gas in an interferometer was investigated theoretically. The splitting-recombination regions were here treated by assuming that the physics of the splitting could be incorporated in boundary conditions of the quantum dynamics. In Ref. [27], the influence of weak nonlinearity on the merging of split atomic gases was investigated and shown to cause an instability of the transfer of population in the guided modes. Recently it was suggested that optical lattices could be used to transport atoms through split potential structures in order to achieve entanglement of neutral atoms [28].

Propagation of local excitation is also of interest in other physical systems. In communication technology, the progress of an optical pulse through guiding structures serves as the model system for both communication and computation applications. In femtosecond laser-induced dynamics of molecular processes, we have a well established area of research, where vibrational states propagate along electronic potential surfaces.

In confinement to atomic waveguide geometries with transverse dimensions around or below micrometer scales, the quantum nature of the atoms starts to dominate the dynamics. Propagation in such potentials will take place in the form of matter waves, we are in the regime of atom optics, and the phenomena can be used to explore new features involving the fundamental properties of quantum dynamics. Work is in progress to create the atom optical analog of standard optical components relying on waveguide structures. This development is partially spurred by the possibili- 
ties to utilize matter-wave guides for quantum logic [29] using controlled collisions of neutral atoms [30].

When the essential physical phenomena are of short duration, they are best understood in the time domain [31]. This is true even when the Hamiltonian contains no time dependence. To simulate realistic systems, one needs to consider many degrees of freedom, which makes the numerical work difficult and in many cases well beyond the resources of even the most powerful modern computers. Due to the exponential growth of Hilbert space with the number of degrees of freedom, well known in quantum information theory, the amount of data to be processed increases exponentially with the number of dimensions. Any method which reduces this number is extremely interesting and will find uses in diverse areas. The use of separated channels or modes offers such an approach, but this requires certain adiabaticity assumptions to hold. In the lowest approximation, the center of the wave packet may be assumed to progress along the classical minimum of the potential valley. The transverse curvature is taken to provide a confining potential around this.

In our previous works, the limitations due to mode coupling were examined both for single waveguides [32] and split potentials [33], and it was shown that the breakdown of adiabatic following of the transverse modes was connected to the intrinsic diffraction of matter waves. The connection with diffraction was shown also for the transition from adiabatic propagation to free expansion in Ref. [34]. The occurrence of reflection of guided matter waves was investigated both for single guides [35] and for split potentials [33]. In both cases considerable backscattering was found to occur in the adiabatic limit and in the case of single guides also in the transition to free expansion. In this work, we consider the channeled splitting of wave packets on two-dimensional potential-energy surfaces, the highest dimensionality comfortably available for numerical work at present. The propagating state is assumed initially to be localized closely along a single minimal path and subsequently propagated through a split of the guiding structure into two identical waveguides. An adiabatic basis of discrete eigenstates is used to reduce the complexity of the problem by reducing the timedependent Schrödinger equation to a set of equations for closely coupled channels. When the physical parameters of the problem allow adiabatic propagation, the discrete basis states decouple, making it possible to model the system as a number of independent one-dimensional wave packets propagating in their respective potential structures. As such the model could apply to as well chemical reaction pathways and optical fiber communication [36] as mesoscopic structures [37], but we essentially have in mind the recently developed microstructures for confined atomic wave packets.

Interferometry depends on the splitting and recombination of matter waves together with local manipulations of the propagating states. It is thus important to understand the physics of matter-wave splitting in terms of localized states. Here we describe the dynamics using a unitary transformation acting on the propagating modes in order to create a basis with suitable localization properties. The formal framework used here is in many respects similar to hybridization in molecular physics [38] which is used to create localized electronic states. Propagation on potential surfaces with multiple minimal valleys also occurs in reactive scattering and many similarities between interferometric devices and branching in chemical reactions exist.

The question whether or not the longitudinal extent of a propagating quantum wave packet will give rise to any observable consequences has caused debate at times. Both experimental results utilizing neutrons [39] and theoretical arguments [40] in favor as well as criticism pointing to alternative interpretations [41] have appeared in the literature. We consider here the influence of longitudinal localization on the physics of wave packets propagating through split potential structures. This is in contrast to our earlier work [33] where the splitting was investigated solely in terms of the transverse eigenstates in order to derive adiabaticity criteria for propagating states. Here we wish to extend the adiabatic propagation schemes to include situations describing typical matter-wave interferometers. Due to the difference in the effective propagation equations, the physics around the splitting point is nontrivial also for coherent superpositions of different propagating modes.

The structure of the paper is as follows: Section II describes the system investigated and the separation of the wave function into distinct modes using transverse eigenstates of the potential-energy surface. In Sec. III, we describe a framework for adiabatic propagation in terms of localized modes obtained using a unitary transformation of the transverse eigenstates. Section IV discusses the case of propagation of coherent superpositions of transverse eigenstates and the influence of finite longitudinal wave-packet size. Finally, Sec. V summarizes the results of the paper and discusses the consequences for interferometric experiments and simulations thereof.

\section{ADIABATIC PROPAGATION}

Matter waves in potential microstructures evolve according to the time-dependent Schrödinger equation. In this work we study a two-dimensional system with stationary potential

$$
i \frac{\partial \Psi(x, y, t)}{\partial t}=-\frac{1}{2} \nabla^{2} \Psi(x, y, t)+V(x, y) \Psi(x, y, t) .
$$

Units are taken to be dimensionless unless otherwise stated; for further details on the consequences of this choice see, e.g., Refs. [32,34]. In the cases of interest here, the propagation will be along the minimal valleys of the potential-energy surface, which we can take to foliate into a continuum of transverse, bound one-dimensional potentials. We then assume that the time-independent Schrödinger equation can be solved in the transverse direction at each point along the minimal curve in order to obtain a set of eigenfunctions:

$$
E_{n}(x) \eta_{n}(x, y)=-\frac{1}{2} \frac{\partial^{2} \eta_{n}(x, y)}{\partial y^{2}}+V(x, y) \eta_{n}(x, y)
$$

The transverse Schrödinger equation (2), which constitutes a Sturm-Liouville system, has a complete set of eigenfunctions $\left\{\eta_{n}(x, y)\right\}$. These can be used in an ansatz together with a set 
of longitudinal wave functions $\left\{\varphi_{n}(x, t)\right\}$ yet to be determined. As these are taken to depend also on time, they determine the time evolution, both propagation along the minimal valley and the confined dynamics in the transverse direction. The total wave function is given by the expansion

$$
\Psi(x, y, t)=\sum_{n=0}^{\infty} \varphi_{n}(x, t) \eta_{n}(x, y)
$$

We insert Eqs. (2) and (3) into Eq. (1), multiply by $\eta_{m}(x, y)$, and integrate over the transverse coordinate, which is thus eliminated by the orthonormality of the basis set. We obtain the expression

$$
\begin{aligned}
i \hbar \frac{\partial \varphi_{m}}{\partial t}= & -\frac{1}{2} \frac{\partial^{2} \varphi_{m}}{\partial x^{2}}+E_{n}(x) \varphi_{m}+\sum_{n=0}^{\infty} A_{n m}(x) \frac{\partial \varphi_{n}}{\partial x} \\
& +B_{n m}(x) \varphi_{n},
\end{aligned}
$$

where we have introduced the first order (or kinetic) couplings

$$
A_{n m}(x)=-\int_{-\infty}^{\infty} \eta_{n}(x, y) \frac{\partial \eta_{m}(x, y)}{\partial x} d y
$$

and the second-order (or potential) couplings

$$
B_{n m}(x)=-\frac{1}{2} \int_{-\infty}^{\infty} \eta_{n}(x, y) \frac{\partial^{2} \eta_{m}(x, y)}{\partial x^{2}} d y
$$

Couplings (5) introduce velocity-dependent interactions. We have here assumed that the coordinate line given by $y=0$, i.e., the origin of the transverse coordinate, does not curve as this will introduce further couplings between the different modes. Propagation of the longitudinal modes is governed by Eqs. (4), a system of coupled one-dimensional Schrödinger equations; these can in principle be solved directly, but in practice this procedure becomes cumbersome as it relies on solving the transverse problem and using the solutions thus obtained to calculate the coupling coefficients (5) and (6). In simplified treatments of matter waves in guiding potential structures, frequently only the ground state is included and the couplings to higher modes are neglected. Here we consider the propagation of wave packets guided close to the minima of a symmetric double-well (SDW) potential

$$
V_{D W}(y, d(x))=\frac{1}{2} \omega_{0}^{2}\left\{y^{2}+d(x)^{2}-2 y d(x) \tanh \left[\omega_{0} y d(x)\right]\right\},
$$

where $d(x)$ is a parameter which determines the distance between the two minima, here taken to depend on the longitudinal coordinate $x$. This potential allows us to determine the ground state exactly. At constant longitudinal coordinate, the transverse ground state for this potential is given by a sum of two identical Gaussians, each centered close to the minima of the two wells:
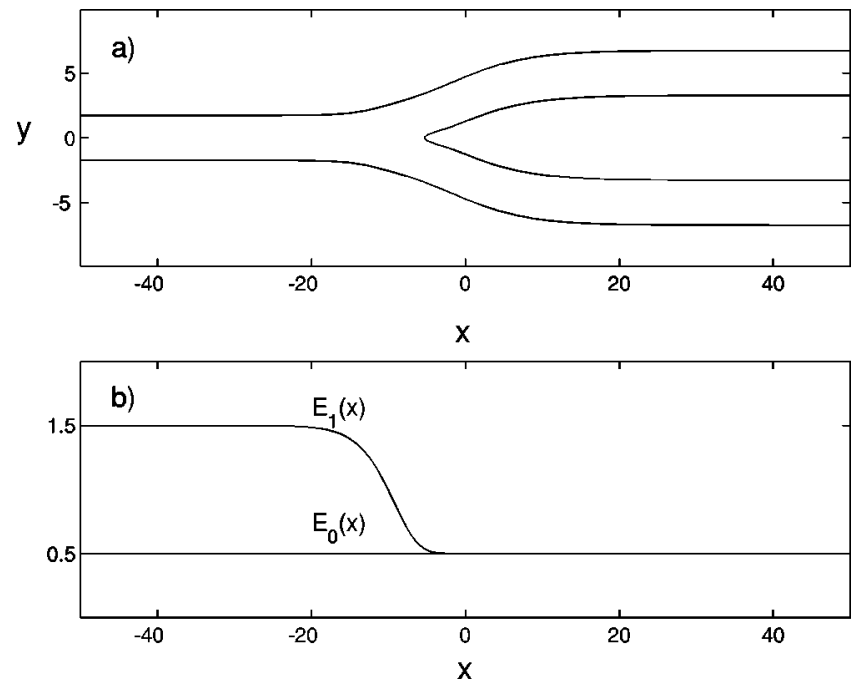

FIG. 1. In (a) a contour line of the potential given by Eqs. (7) and (9) with the parameters given by $X=10, \omega_{0}=1, d_{\max }=5$ is shown. The chosen equipotential level corresponds to distances twice the ground-state width away from the bottom of the wells. In (b) the transverse binding energies for the first two states are shown as functions of longitudinal distance. We see that the two transverse eigenenergies become nearly degenerate shortly before $x=0$, resulting in a markedly increased tunneling time between the two wells. Units are dimensionless.

$$
\begin{aligned}
\eta_{0}(y, d(x))= & \frac{1}{\sqrt{2\left(1+e^{-\omega_{0} d(x)^{2}}\right)}}\left(\frac{\omega_{0}}{\pi}\right)^{1 / 4} \\
& \times\left[e^{-\left(\omega_{0} / 2\right)[y+d(x)]^{2}}+e^{-\left(\omega_{0} / 2\right)[y-d(x)]^{2}}\right]
\end{aligned}
$$

and the ground-state energy is constant, $E_{0}(d(x))=\frac{1}{2} \omega_{0}$. In order to investigate the behavior of wave packets in SDW potentials the distance function was chosen as

$$
d(x)=\frac{1}{2} d_{\max }\left[1+\tanh \left(\frac{x}{X}\right)\right]
$$

where $d_{\max }$ is half the asymptotic distance between the minima and $X$ is a parameter which controls the extension of the region where the transition from a single guide to a double guide takes place. Twice the function $d(x)$ strictly speaking only equals the distance between the minima for $d=0$ and in the asymptotic limit of large separations. In Fig. 1 the potential given by Eqs. (7) and (9) with the parameters $X=10, \omega_{0}=1$, and $d_{\max }=5$ is shown together with the transverse binding energies for the two lowest states. These two states become degenerate with respect to energy for large interwell distance function $d(x)$. The behavior of the transverse eigenstates as functions of longitudinal distance can be seen in Fig. 2, where the same parameters as in Fig. 1 were used.

In Fig. 3 the magnitudes of couplings (5) and (6) are shown for the two lowest-energy eigenstates of the SDW potential used here. For a symmetric potential, the transverse eigenstates have definite parity. It is easily seen that taking the derivative with respect to the longitudinal coordinate pre- 

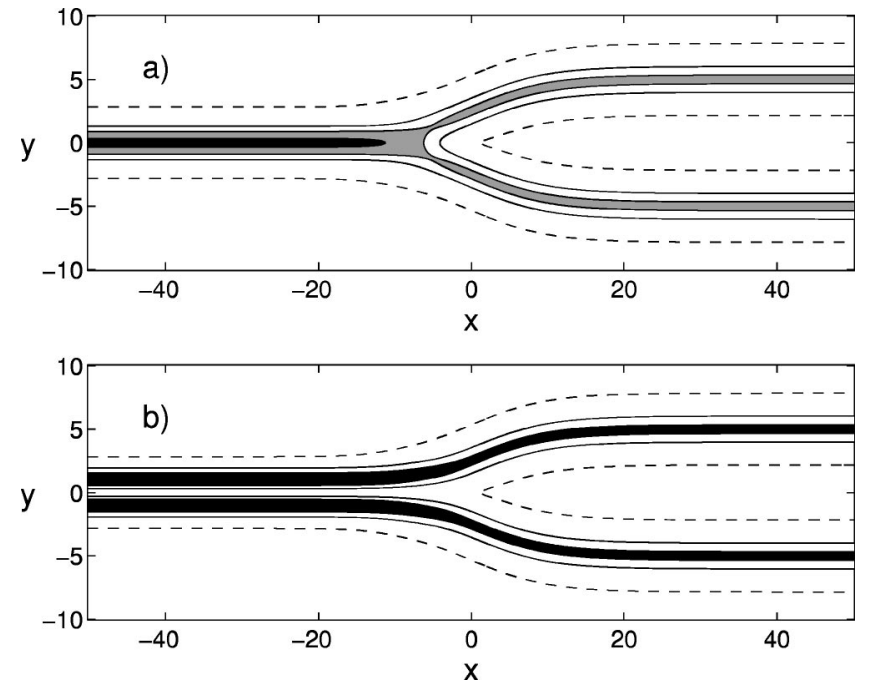

FIG. 2. Mode structure of the two lowest-energy eigenstates (a) $\eta_{0}(y, d(x))$ and (b) $\eta_{1}(y, d(x))$. Shown is the probability density for the two transverse modes as function of both transverse and longitudinal coordinates. The two modes are seen to split into two distinct pieces each centered in one outgoing guide. Axes are in dimensionless units.

serves parity of the transverse states. As a consequence, the coupling matrix elements $A_{i j}(x)$ and $B_{i j}(x)$ vanish for transitions mixing odd and even states.

In our previous work [33], adiabatic propagation in the SDW potential given by Eqs. (7) and (9) was investigated. The magnitude of the nonadiabatic excitation, measured as probability transferred from the ground state to excited states during propagation through the splitting region, was found to depend on a single dimensionless parameter, the Fresnel number, named after the analogous quantity in classical optics [42]. Small Fresnel numbers correspond to the far-field zone, where the transverse distribution is determined by the contribution from a single Fresnel zone only. For a wave packet propagating in a splitting potential, the equiprobability lines traced out during the evolution will diverge and, given that the widening is not too rapid, adjustment to the new width will take place. Taking the potential to be changing over distances of the order of $L$, the Fresnel number for this situation is given as

$$
N=\frac{z_{R}}{L}=\frac{1}{L \lambda \omega_{0}}
$$

where $\lambda$ denotes the de Broglie wavelength corresponding to the longitudinal motion. Adiabatic propagation of wave packets in potential structures will take place when the changes are such that the Fresnel parameter is small. Thus the question is whether or not the changes occur over a length scale comparable to or larger than the Rayleigh length, which is the length a suddenly released beam requires in order to evolve into diverging at a constant angle determined by its momentum distribution. The length scale $L$ in the expression for the Fresnel parameter is, in classical, optics the distance over which a beam diffracts in width by

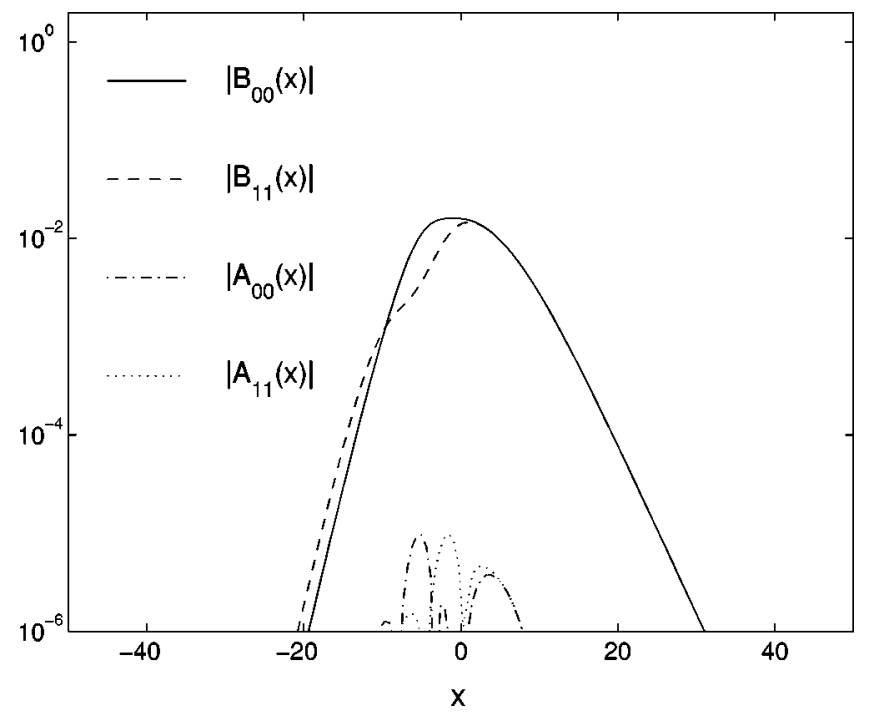

FIG. 3. Absolute values of the couplings given by Eqs. (5) and (6) for the ground and first excited eigenstates. The relevant energy scale is set by the transverse oscillator frequency $\omega_{0}=1$, i.e., the values are to be compared with unity. The velocity-dependent couplings $A_{00}(x)$ and $A_{11}(x)$ are seen to be very small for the momentum values needed for adiabatic propagation, whereas the couplings $B_{00}(x)$ and $B_{11}(x)$ are a few percent of the ground-state energy. Units are dimensionless.

about a factor of $\sqrt{2}$. Here a suitable choice is the distance over which the interwell distance separates to about

$$
d\left(x_{u}\right)=\sqrt{2} \Delta y=\sqrt{2 \omega_{0}},
$$

where $\Delta y$ denotes the width of transverse ground state. This is also the point, in the longitudinal direction, at which the central barrier height equals the ground-state energy. As a result, an assembly of classical particles with the same distribution of initial positions and momenta would here see a bifurcation in phase space. The value given by Eq. (11) alone does not suffice to estimate the length scale and a second point must be pinned down using some criterion of the type

$$
d\left(x_{l}\right) \geq \Delta y .
$$

It turns out that the choice of lower end point is fairly insensitive to the exact details of criterion (12), probably due to the exponential character of the distance function. A suitable value is given by the point where distance between the wells was larger than about 1.001 times the ground-state width. The distance for potential changes is thus taken to be

$$
L=x_{u}-x_{l},
$$

where $x_{u}$ and $x_{l}$ are defined above. With this choice of the length scale, the nonadiabatic excitation probability has been found to be a function of the Fresnel parameter only, regardless of the values of other physical parameters [33]. 


\section{LOCALIZED PROPAGATION}

In our earlier works, the investigation of adiabatic propagation was mainly focused around changes related to the diffractive spreading of matter-wave beams, this being equivalent to the case of wave packets propagating in potential structures of changing width [32]. For a potential-energy surface with a single minimal path, the idea of separation of the wave function is in principle unproblematic as the longitudinal modes propagate close to the minima.

For the case of splitting, considered here, such an approach neglects the process of dividing the transverse states into two separate halves by the raising of a central barrier. As the incoming transverse oscillator frequency in the potential used here coincides with the ones in the two outgoing guides, an alternative picture could be envisioned. It is possible to consider the incoming wave packet as being a superposition of two almost identical ones each to be guided along one of the two degenerate minima. In this case there are two approximately independent wave packets propagating in two waveguides curving in different directions away from each other. Nonadiabatic excitations would then be regarded as arising due to the longitudinal curvature of each guide.

For the case of symmetric potentials with split minima the transversally localized propagating modes are the transverse energy eigenstates of the binding potential in the region before the splitting. When the potential minima are far apart, the localized states are known to be given by linear combinations of pairs of energy eigenstates according to

$$
\eta_{L / R}(y, d(x))=\frac{1}{\sqrt{2}}\left[\eta_{n}(y, d(x)) \pm \eta_{n+1}(y, d(x))\right],
$$

where the transverse quantum number $n=0,2,4, \ldots$ An adiabatic propagation scheme thus has to deal with the apparent conflict of continuously guiding energy eigenstates, which have definite parity and are localized in a single minimum, into states that are delocalized, when it is known that wave equations have localized propagating solutions. For the case of large constant separation between the waveguides, the propagation, Eq. (4), becomes separated into independent equations since the couplings depend on the spatial variations of the transverse eigenstates. As, in addition, the energy levels become pairwise degenerate, the propagation equations remain invariant under unitary transformations among the pairs of eigenstates. Two natural choices for basis states are transverse energy eigenstates or localized states. The latter will be the preferable choice whenever the individual potential wells are disturbed in a local fashion, for instance in an interferometric setup, as the influence from the other well is negligible.

The condition for adiabaticity in quantum dynamics is often formulated as a requirement that the internal time scale, in this case the transverse oscillation time

$$
T_{o s c} \sim 1 / \omega
$$

should be shorter than the time scale under which the Hamiltonian undergoes considerable changes, here denoted by $\Delta t$. For wave-packet dynamics in potential structures, the exter- nal time scale has to be estimated with great care or else the criterion might become a poor indicator of adiabatic evolution. For wave-packet dynamics in spatially changing potential structures, the diffraction criterion can be used as a means to estimate the allowable changes in a less ad hoc manner. In addition to the two time scales mentioned, the system under consideration here contains a third, the transverse tunneling time given by the energy split between ground and first excited states,

$$
T_{\text {tunn }} \sim 1 / \Delta E
$$

a function of the distance between the wells and the transverse oscillator frequency. As the ground-state energy is constant in the double-well potential chosen, the tunneling time is always larger than the transverse oscillation time:

$$
T_{\text {osc }} \leqslant T_{\text {tunn }}
$$

Depending on the relative sizes of the three time scales, we can distinguish between different regimes. First, if the external time scale is the shortest,

$$
\Delta t \ll T_{o s c} \leqslant T_{\text {tunn }}
$$

the dynamics will be nonadiabatic resulting in sizable excitation of higher transverse modes. The potential changes occur fast enough to result in the wave packet propagating with constant shape through changes. On times scales of the order of the oscillation time, the resulting changes start to be noticeable when transverse oscillations induced by the nonadiabatic transitions become visible. If both the oscillation and tunneling times are shorter than the external time scale,

$$
T_{\text {osc }} \leqslant T_{\text {tunn }} \ll \Delta t
$$

the potential changes over length scales larger than the Rayleigh length and the dynamics evolves adiabatically with respect to the transverse eigenstates. Propagating wave packets thus behave as if composed of a single mode, although they might be split into two partially overlapping halves. For large separations, the tunneling time approaches infinity rapidly, as the two lowest states become degenerate in energy, and it might become larger than the external time scale:

$$
T_{\text {osc }} \ll \Delta t \ll T_{\text {tunn }} .
$$

In this case, transverse energy eigenstates will not be good adiabatic states that are followed, but the localized states, which to a very good approximation are single-well ground states, propagate adiabatically and couple weakly to the neighboring well only through the overlap which decays exponentially with the distance between the wells. To relate this with the adiabaticity criterion (10) from our earlier work $[32,33]$ we first note that the time scale for the potential change can be estimated as

$$
\Delta t \approx \frac{L}{k_{x}} \propto L \lambda,
$$




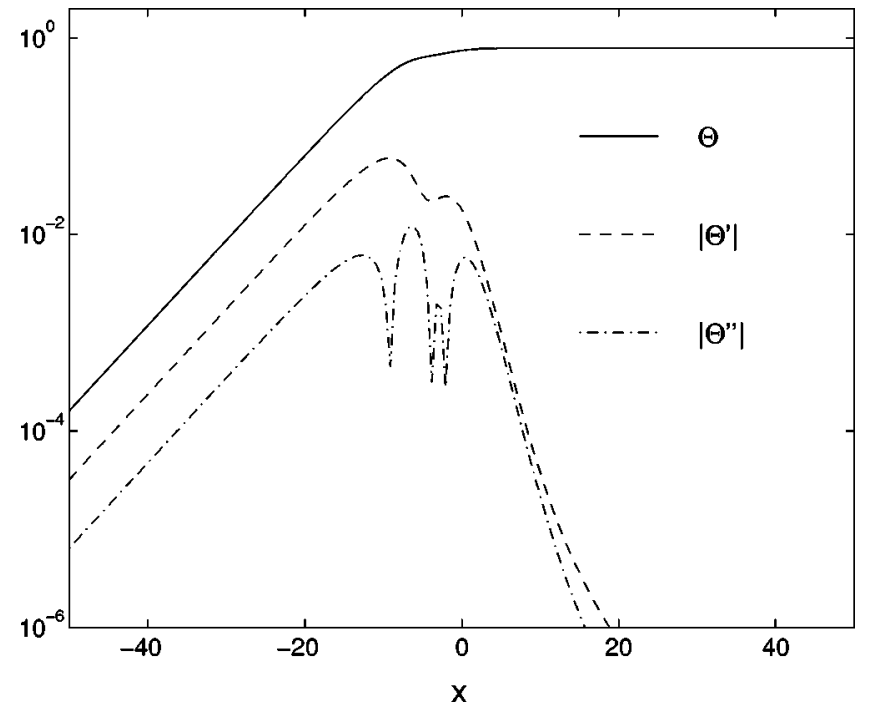

FIG. 4. Spatial variation of the transformation angle $\theta(x)$ for the SDW potential given by Eqs. (7) and (9) with the same parameter values as for Fig. 1. Shown dashed is the first derivative and dotdashed the second derivative with respect to the longitudinal coordinate. The potential parameters are the same as in Fig. 1. Axes are in dimensionless units.

where the length $L$, both for single guides and potential splittings, is related to the transverse change of the guided state and has to be chosen as described in Sec. II. To estimate the tunneling time (16), we use the fact that the energy difference between the first two transverse states approximately was found to be

$$
\Delta E \approx \omega_{0} \exp \left(-2 \omega_{0} d^{2}\right)
$$

Using Eqs. (21) and (22) in Eq. (20), we find

$$
\exp \left(-2 \omega_{0} d^{2}\right) \ll N \ll 1
$$

where $N$ is the Fresnel parameter as defined by Eq. (10). If both conditions in Eq. (23) are met, then the error due to nonadiabatic excitations will be limited by the intrinsic diffraction of matter waves as described in Ref. [32]. As the well separated guides may each go through very different evolution, it is not meaningful to talk about the transverse eigenstates of the total potential as being the adiabatic states that are followed. Problematic situations emerge, for instance, in the case of differing path lengths between the guides in a Mach-Zender interferometer or propagation at right angles or even backwards after a $180^{\circ}$ bend.

In order to construct a localized propagation scheme for the two lowest transverse states, we take our modes to be linear combinations depending on the longitudinal coordinate. In order to preserve orthonormality, we choose the states to be transformed to the new localized basis through a unitary transformation:

$$
\left[\begin{array}{l}
\eta_{L}(x, y) \\
\eta_{R}(x, y)
\end{array}\right]=\left[\begin{array}{cc}
\cos \theta & -\sin \theta \\
\sin \theta & \cos \theta
\end{array}\right]\left[\begin{array}{l}
\eta_{0}(y, d(x)) \\
\eta_{1}(y, d(x))
\end{array}\right],
$$
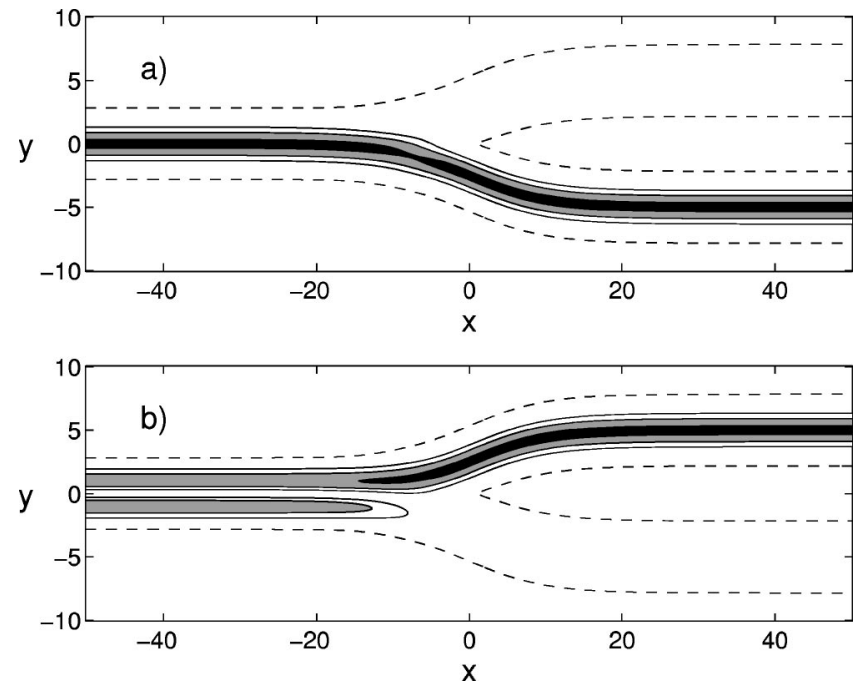

FIG. 5. Mode structure of the localized mode functions (a) $\eta_{L}(x, y)$ and (b) $\eta_{R}(x, y)$, here shown as the respective probability densities. Each mode function is seen to be centered on the single guide before the split. After the split each mode has only a small probability of being in one of the outgoing guides, thus being predominantly localized to the other. For the mode $\eta_{R}(x, y)$, which before the split is equal to the first excited state, the node seems to disappear around the splitting region. This is not true, however, as the mixing of the two energy eigenstates causes each localized mode to necessarily have a node in the outgoing distributions. Axes are in dimensionless units.

where the transformation angle $\theta(x)$ is chosen in such a way that the new basis states at all positions along the waveguide are localized in each well in the sense that the expectation values of the transverse coordinate taken with respect to either state will equal the positions of the two Gaussians which constitute the ground state

$$
\left\langle\eta_{L / R}|y| \eta_{L / R}\right\rangle=\mp d(x),
$$

where the signs were chosen in order to localize $\eta_{L}$ in the left minimum, i.e., at negative transverse coordinate values. This corresponds to the lower well in Figs. 1 and 2. Using Eq. (24) in Eq. (25) we arrive at

$$
\theta(x)= \pm \frac{1}{2} \arcsin \left(\frac{d(x)}{y_{01}}\right),
$$

where $y_{01}$ denotes the dipole matrix element between $\eta_{0}$ and $\eta_{1}$. The spatial variation of the transformation angle $\theta(x)$ can be seen in Fig. 4. For small values of separation $d_{\max }$ the angle $\theta$ will be close to zero whereas for large separations it will approach $\pi / 2$ as the localized states approximately will be given by Eq. (14). The structure of the localized modes can be seen in Fig. 5 for the split potential used in this work. For the mode which equals the transverse ground state for large negative values of the longitudinal coordinate there is little change in the transverse size or other properties along the propagation direction. For the other mode, however, the node around $y=0$ seems to disappear as the transverse shape after the splitting is close to that of a Gaussian localized in 
the upper minimum. This change occurs just before $x=0$ as the upper half of the eigenstate is seen to grow into dominating the mode. The total wave function is now given by

$$
\begin{aligned}
\Psi(x, y, t)= & \eta_{L} \varphi_{L}+\eta_{R} \varphi_{R}=\eta_{0}\left[\varphi_{L} \cos \theta+\varphi_{R} \sin \theta\right] \\
& +\eta_{1}\left[-\varphi_{L} \sin \theta+\varphi_{R} \cos \theta\right] .
\end{aligned}
$$

We thus see that writing the total wave function as a linear combination of two localized transverse modes is equivalent with transforming the longitudinal modes according to

$$
\left[\begin{array}{l}
\varphi_{0}(x, t) \\
\varphi_{1}(x, t)
\end{array}\right] \rightarrow\left[\begin{array}{l}
\varphi_{L}(x, t) \\
\varphi_{R}(x, t)
\end{array}\right]=\left[\begin{array}{cc}
\cos \theta & \sin \theta \\
-\sin \theta & \cos \theta
\end{array}\right]\left[\begin{array}{l}
\varphi_{0}(x, t) \\
\varphi_{1}(x, t)
\end{array}\right] .
$$

If we now use ansatz (28) in the time-dependent Schrödinger equation and project away the dependence on the transverse coordinate, we arrive at the propagation equations for the localized modes

$$
\begin{aligned}
& i \frac{\partial \varphi_{L}(x, t)}{\partial t}=-\frac{1}{2} \frac{\partial^{2} \varphi_{L}(x, t)}{\partial x^{2}}+E_{L}(x) \varphi_{L}(x, t)+W \varphi_{R}(x, t), \\
& i \frac{\partial \varphi_{R}(x, t)}{\partial t}=-\frac{1}{2} \frac{\partial^{2} \varphi_{R}(x, t)}{\partial x^{2}}+E_{R}(x) \varphi_{R}(x, t)+W \varphi_{L}(x, t),
\end{aligned}
$$

where the potentials and the off-diagonal coupling are given by

$$
\begin{gathered}
E_{L}(x)=\cos ^{2} \theta\left[E_{0}(x)+B_{00}(x)\right]+\sin ^{2} \theta\left[E_{1}(x)+B_{11}(x)\right], \\
E_{R}(x)=\sin ^{2} \theta\left[E_{0}(x)+B_{00}(x)\right]+\cos ^{2} \theta\left[E_{1}(x)+B_{11}(x)\right], \\
W(x)=\sin \theta \cos \theta\left[E_{0}(x)+B_{00}(x)-E_{1}(x)-B_{11}(x)\right] .
\end{gathered}
$$

We have here neglected the spatial variation of the transformation angle $\theta(x)$ in that its derivatives with respect to the longitudinal coordinate $x$ have been dropped. The dominating off-diagonal term is determined by $W(x)$ in Eq. (30) and this can be considered to give the coupling matrix elements to lowest order in the spatial derivatives of $\theta(x)$. In Fig. 4 the spatial variation of the transformation angle and its first and second derivatives are shown. The propagation equations for localized modes in a split potential thus have the form of a two-level system with spatially dependent energies, couplings, and velocity-dependent interactions. For large separations of the two minima, the level splitting rapidly goes to zero and all couplings can be neglected. When the distance function $d(x)$ is close to zero, the couplings are also negligible and the propagation is in the form of two independent modes. The framework defined here thus models the splitting of matter waves using basis states which are localized both in the single-guide region and in the double-guide region. In the splitting region localized propagation necessitates coupling between the two modes. For a quantitatively more accurate treatment, the terms dependent on the derivatives of the transformation angle must also be taken into account. In Fig.

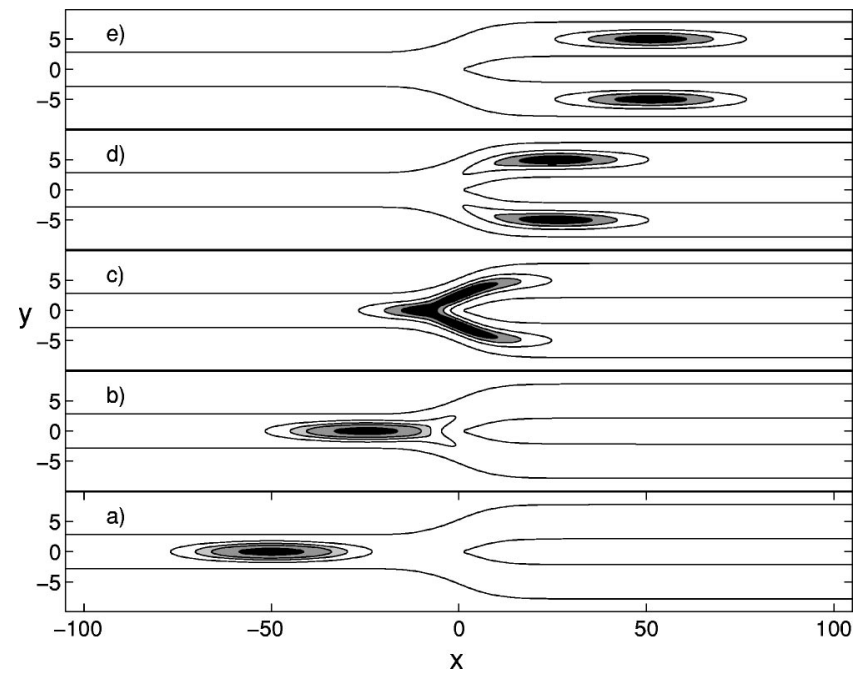

FIG. 6. Snapshots of a wave packet initially in the ground-state mode $\eta_{0}(x, y)$. Pictures (a)-(e) show the probability density for a wave packet initially in the ground state at instants separated by equal amounts of time. As time progresses the wave packet propagates through the split and enters the asymptotic region beyond the split. From the final snapshot (e) it is seen that the probability is distributed in a symmetric manner between the outgoing guides. The initial wave packet was a Gaussian of width $\Delta x=20$ and momentum $k_{x}=1$. Units are dimensionless.

6 , snapshots of a wave packet initially in mode $\eta_{L}(x, y)$ which for large negative distances equals the ground state, are shown during propagation through a splitting region. Without any interaction between the two modes, the wave packet will be guided completely into the lower outgoing guide, completely unphysical result as the ground state mode is split equally between the two degenerate wells, as can be seen from Fig. 2. At the splitting, the interaction leads to the creation of a component in mode $\eta_{R}(x, y)$, which is channeled into the other guide. The dynamics for both modes is shown in Fig. 7. It is seen here that although the wave packet is initially completely in the mode $\eta_{L}(x, y)$, the interaction around $x=0$ causes a significant growth of probability in the other mode $\eta_{R}(x, y)$. For ground-state propagation, the expected result would be an equal split between the outgoing modes resulting in a probability transfer of $\Delta P=0.5$ regardless of the longitudinal momentum $k_{x}$. Any deviations from this result come from numerical inaccuracies in the wavepacket propagation, failure to include enough couplings, or limited accuracy in the calculation of the coupling coefficients (5) and (6).

\section{COHERENT SUPERPOSITIONS}

A fundamental law of quantum mechanics is the superposition principle, which can give rise to interesting new dynamics through the interference of quantum states. The linear combination given by Eq. (14), i.e., an equal superposition of two energy eigenstates, corresponds for the case of far separated potential wells to a localized state. Here we wish to investigate the dynamics of superposition states with limited spatial extent in the longitudinal direction, and take the ini- 

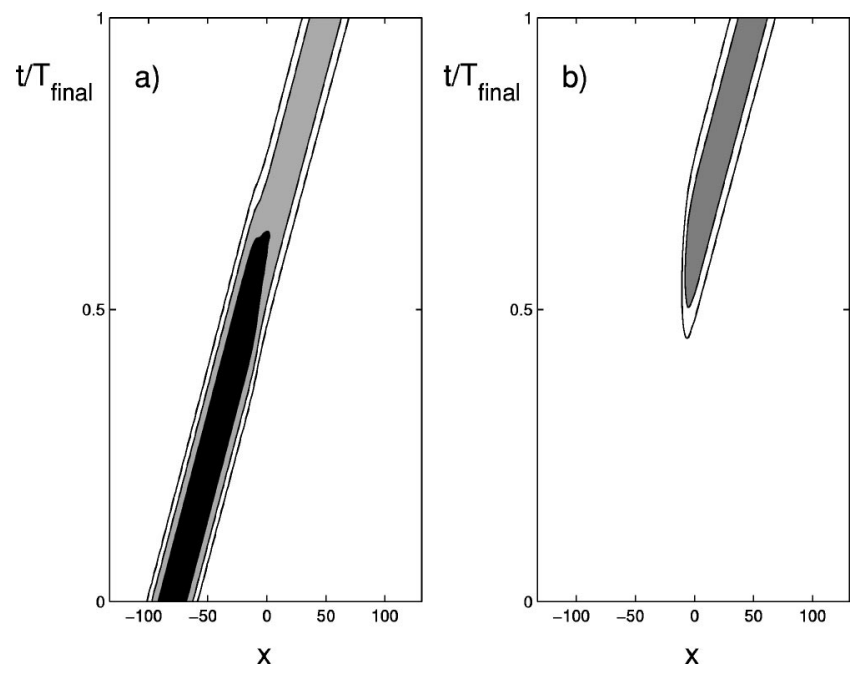

FIG. 7. Probability density of the localized modes propagating through the potential shown in Fig. 1. In (a) $\left|\varphi_{L}(x, t)\right|^{2}$ is shown, and in (b) $\left|\varphi_{R}(x, t)\right|^{2}$. The wave packet was initially in the groundstate mode. In the asymptotic region before the split the wave packet is thus almost completely in $\varphi_{L}(x, t)$, which is guided to the lower well in Fig. 5. It is seen that considerable interaction between the localized modes takes place, and that the final distribution is symmetric as expected. Axes are in dimensionless units.

tial state to be a general coherent superposition of equal amounts of ground and first excited states,

$$
\Psi=\frac{1}{\sqrt{2}}\left(\eta_{0}+e^{i \Delta \theta} \eta_{1}\right),
$$

where $\Delta \theta$ denotes the relative phase between the states, a quantity that will evolve with time causing the probability distribution to shift in space. The states with extreme localization are given by

$$
\Psi_{L / R}=\frac{1}{\sqrt{2}}\left(\eta_{0} \pm \eta_{1}\right),
$$

i.e., by taking the phase angle to be either $\Delta \theta=0$ or $\Delta \theta$ $=\pi$. If we calculate the expectation value of the transverse coordinate we have

$$
\begin{aligned}
\langle y\rangle & =\langle\Psi|y| \Psi\rangle \\
& =\left\langle\frac{1}{\sqrt{2}}\left(\eta_{0}+e^{i \theta} \eta_{1}\right)|y| \frac{1}{\sqrt{2}}\left(\eta_{0}+e^{-i \Delta \theta} \eta_{1}\right)\right\rangle \\
& =y_{01} \cos (\Delta \theta),
\end{aligned}
$$

where $y_{01}$ denotes the dipole matrix element between the ground and first excited states, a quantity that approaches half the interwell distance for the case of well separated potential minima.

For a superposition wave packet of negligible extent in the longitudinal direction, the probability to go to the left or to the right will be given by the projection of the initial state onto transverse states localized in either well,

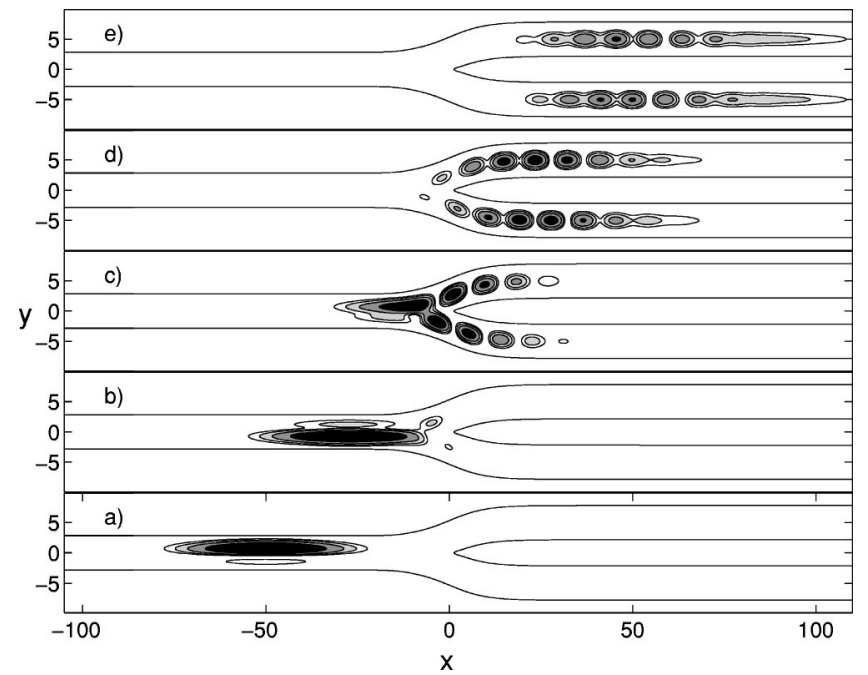

FIG. 8. The adiabatic splitting of a wave packet being transversally a coherent superposition of the lowest two eigenstates. As this state oscillates from side to side in the waveguide, with a period given by the transverse binding potential, one would naively expect the outgoing state to be localized in either outgoing guide given that the initial phase of the components has been appropriately tuned. On the contrary it is seen that the wave packet is fractioned into smaller portions each centered in one of the guides and propagating outwards. This behavior is due to the differing arrival times for different longitudinal sections of extended wave packets. The spatial period of the slicing is approximately given by the distance traveled longitudinally during one transverse oscillation. Units are dimensionless.

$$
\begin{aligned}
p_{L / R} & =\left|\left\langle\Psi \mid \Psi_{L / R}\right\rangle\right|^{2} \\
& =\left|\left\langle\frac{1}{\sqrt{2}}\left(\eta_{0}+e^{i \theta} \eta_{1}\right) \mid \frac{1}{\sqrt{2}}\left(\eta_{0} \pm \eta_{1}\right)\right\rangle\right|^{2} \\
& =\frac{1}{2}[1 \pm \cos (\Delta \theta)] .
\end{aligned}
$$

We thus see that the probability to go to the left or right at the split is dependent on the relative phase between the two energy eigenstates. In the center of mass frame of a quantum particle, well localized in the longitudinal direction, traveling at constant momentum, the phase difference between the two states can be assumed to change over a small time interval according to

$$
d \theta=\Delta E d t \approx \frac{\Delta E}{\frac{\partial x}{\partial t}} d x .
$$

This gives us for the total phase shift during propagation

$$
\Delta \theta=\int_{x_{i}}^{x_{f}} \frac{\Delta E}{\partial x} d x=\frac{C}{k_{x}},
$$

where $x_{i}$ and $x_{f}$ denote initial and final positions in the longitudinal direction and the proportionality constant $C$ is in- 

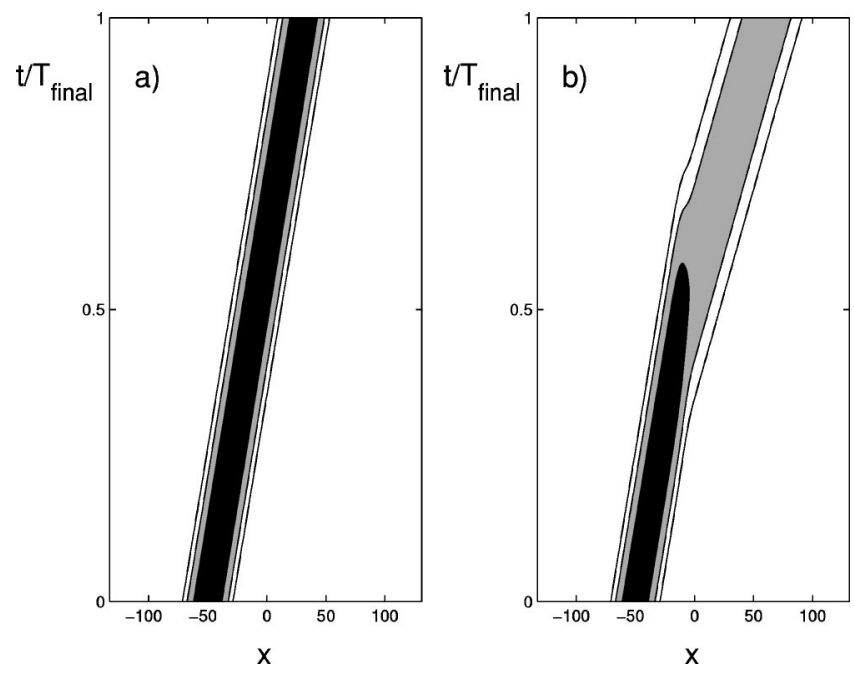

FIG. 9. The adiabatic splitting of a wave packet initially being a coherent superposition of the lowest two transverse eigenstates. The figure shows a density plot of the probability in the propagating eigenstates, $\left|\varphi_{0}(x, t)\right|^{2}$ in (a) and $\left|\varphi_{1}(x, t)\right|^{2}$ in (b), as functions of longitudinal coordinate and time. The oscillations seen in Fig. 8 are absent here as no probability is transferred between the modes in this basis. Axes are in dimensionless units.

troduced for convenience. As the energy split $\Delta E$ goes to zero rapidly after the split, the phase shift slows down, indicating that the oscillation time between the wells, i.e., the tunneling time, becomes much larger than the oscillation period in each well. The dependence on the final position $x_{f}$ in Eq. (36) will thus be negligible given that the propagation has been taken beyond the splitting.

If the initial wave packet is spread out in the longitudinal direction, different parts will reach the split at different times thus having different local values of the relative phase $\Delta \theta$, possibly differing by large amounts. In Fig. 8 we see a coherent superposition wave packet propagating through a split. As the longitudinal momentum is low, a spatially periodic slicing of the wave packet into portions entering different outgoing guides is seen to take place. If several oscillations occur during the split, there will be approximately equal probability for the particle to go in either direction. In Fig. 9 the same splitting is presented as probability density in the transverse eigenstates as functions of time and longitudinal distance. It is seen that both modes propagate forward without noticeable disturbances. The only exception to this is the acceleration of the first excited mode around $x=0$ due to the release of additional energy as the transverse binding energies approach degeneracy. In Fig. 10 the splitting is shown in terms of localized modes in the same way as in Fig. 9. Here, however, in contrast to the previous figure, the periodic slicing of the wave packet is clearly visible in both localized modes. The localized mode picture thus has the ability to reveal information hidden in the relative phases of superposition states.

If we want a wave packet to go into only one of the outgoing guides and, in addition propagate adiabatically, there are several conditions that will have to be fulfilled. First, the wave packet must not conduct a full transverse
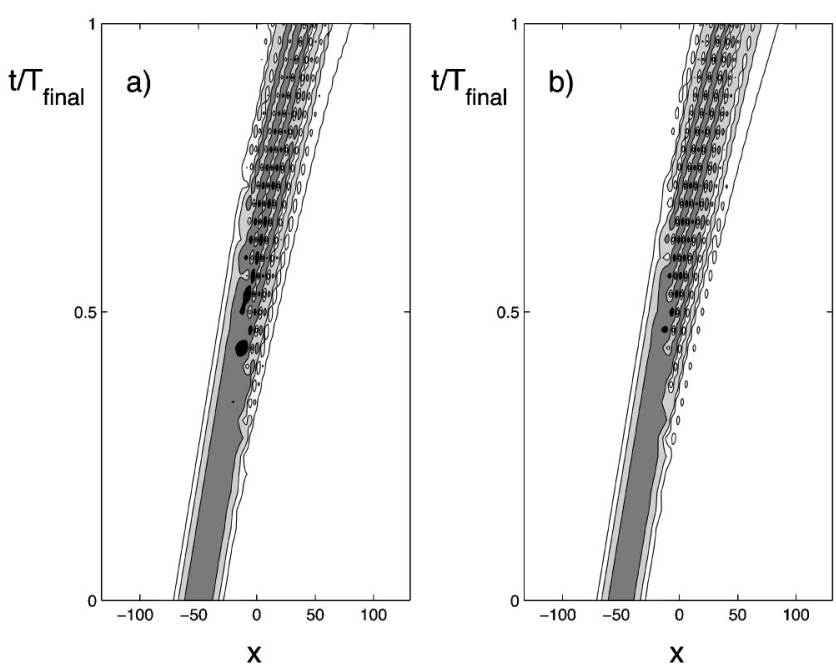

FIG. 10. The adiabatic splitting of a wave packet being initially a coherent superposition of the lowest two transverse eigenstates, as described in terms of the localized modes. The figure shows probability density vs longitudinal coordinate and time (a) for the mode $\varphi_{L}(x, t)$ and (b) for the mode $\varphi_{R}(x, t)$. The coherent oscillations during the splitting of the wave packet are clearly visible here. Units are dimensionless.

oscillation as it propagates a distance corresponding to its longitudinal spread,

$$
2 \frac{\Delta x}{k_{x}} \ll \frac{2 \pi}{\omega_{0}} .
$$

Second, the wave packet should not spread out too much during the split as this will cause the first condition to be broken. We require that the time it takes to propagate through the split should be much smaller than the time of spreading in the longitudinal direction,

$$
\frac{L}{k_{x}} \ll \Delta x^{2}
$$

If the atoms are kept in an optical lattice in the longitudinal direction, as suggested in Ref. [28], requirement (37) is no longer needed as there is no dispersion broadening the distribution. Instead an adiabaticity condition for the longitudinal motion of the optical lattice has to be fulfilled if the atoms are to remain in the localized ground states of the optical lattice. Finally, the propagation should take place with negligible nonadiabatic excitation

$$
\frac{1}{L \omega_{0} \lambda} \ll 1
$$

where $\lambda$ is the longitudinal de Broglie wavelength. Combining Eqs. (37)-(39) we have

$$
\frac{\Delta x}{k_{x}} \ll \frac{1}{\omega_{0}} \ll \frac{L}{k_{x}} \ll \Delta x^{2} .
$$


If all the conditions included in Eq. (40) are fulfilled, we expect the wave packet to exit the splitting region predominantly in only one of the outgoing guides. In order to estimate the amount exiting in the left guide, we calculate an average of $p_{L}(\theta)$ taken over the initial wave packet extended in the longitudinal direction as

$$
P_{L}=\int_{-\infty}^{\infty}|\varphi(x, 0)|^{2} p_{L}(\theta(x)) d x,
$$

where the intial probability distribution is taken to be Gaussian,

$$
|\varphi(x, 0)|^{2}=\frac{1}{\sqrt{2 \pi} \Delta x} \exp \left(-\frac{\left(x-x_{0}\right)^{2}}{2 \Delta x^{2}}\right),
$$

with an initial width $\Delta x$ and initial position $x_{0}$. If the initial position is far from the splitting region and the wave packet is sufficiently localized at $t=0$, we take

$$
\Delta \theta(x+X) \approx \Delta \theta(x)+\int_{x}^{x+X} \frac{\Delta E}{k_{x}} d x \approx \Delta \theta(x)+\frac{\Delta E}{k_{x}} X
$$

as an approximate expression for the shift of relative angle with increasing distance from the splitting region. Using Eqs. (34), (42), and (43) in Eq. (41), we can calculate the fraction of the wave packet, averaged over the initial distribution, exiting through the left guide after propagation as

$$
P_{L}=\frac{1}{2}\left[1-\exp \left(-\frac{1}{4} \frac{\Delta x^{2} \omega_{0}^{2}}{k_{x}^{2}}\right) \cos \left(\frac{C}{k_{x}}\right)\right],
$$

where the constant $C$ is determined by Eq. (36) with $x_{i}$ $=x_{0}$, in principle. In practice it turns out that the total phase is not given by a simple expression of this kind probably due to the nonuniform acceleration in the longitudinal direction around the splitting, which was ignored in the derivation of Eq. (44). In the discussion above, adiabaticity was implicitly assumed and the longitudinal spreading during propagation neglected. As a result, the maximal transverse localization into the outgoing guides depends, apart form an oscillatory factor, on the parameter combination

$$
\frac{\Delta x \omega_{0}}{k_{x}} \ll 1 .
$$

This condition is also given by Eq. (37). This shows that localization in either outgoing matter-wave guide for a wave packet of finite longitudinal extent is determined by the number of transverse oscillations that are completed during passage through the splitting region.

This was tested by propagating wave packets initially prepared in a coherent superposition state through a potential splitting, varying the longitudinal momentum and observing the fraction of the wave packet entering the left (in Fig. 8 the lower) guide. The result is shown in Fig. 11. Open circles show the results from wave-packet simulation and the solid line is given by Eq. (44) where the constant $C$ was used as a

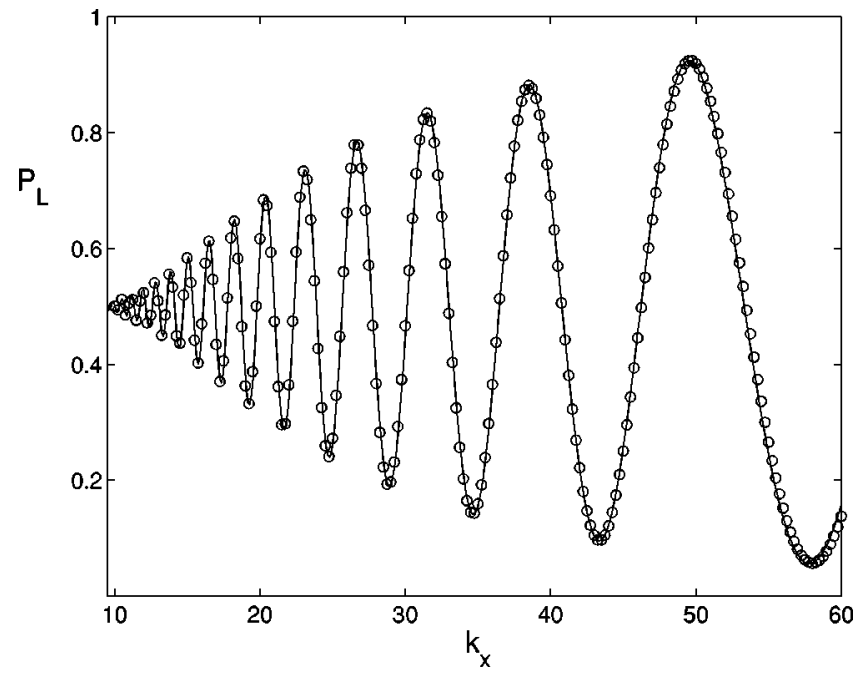

FIG. 11. Probability of finding a wave packet exiting from the left guide as a function of longitudinal momentum. For low momenta, the wave packet is equally split between the output ports, whereas for higher momenta an oscillation can be seen. The amplitude increases with increasing momenta, whereas the oscillation frequency decreases. Open circles denote results obtained from numerical solutions of the time-dependent Schrödinger equation (1) for a potential with parameters $\omega_{0}=1, L=500$, and $k_{x}$ varying. The longitudinal extent of the wave packet was $\Delta x=40$. The parameter $C$ in Eq. (44) has been fitted here.

single fitting parameter. In the derivation above, the longitudinal momentum was taken to be constant during all of the propagation. In reality the excited-state component will be accelerated as the energy difference goes to zero after the split causing an additional phase shift neglected here. The expression given by Eq. (44) agrees well with the numerical results over the whole range of momenta used. For low momenta, there is a probability close to one-half of going into the left guide due to the periodic slicing of the wave packet, as illustrated in Fig. 8. For increasing momenta, the wave packet starts to get split asymmetrically as fewer oscillations take place during the splitting. The probability of going either way shows an oscillatory behavior with increasing amplitude, exactly as predicted by Eq. (44). For the limiting case of infinite longitudinal momentum, which is far beyond the adiabatic approximation, the relative phase between the components of the wave packet does not change at all and is also homogenous along the wave packet. The probability of the wave packet to exit in either of the guides should thus approach a constant value. This region lies beyond the momentum range presented in Fig. 8, and would from a computational point of view be very demanding to probe. We thus see that, given suitable initial wave packets are prepared, propagation through a potential splitting can be used to extract information about the longitudinal structure of matter waves.

\section{DISCUSSION}

We have investigated the propagation of guided matter wave in split potential structures. For well separated 
waveguides it is intuitively appealing to model the dynamics as being in the form of wave packets propagating along independent potential minima. Under these circumstances, the wave packets are expected to be subjected to local adiabaticity conditions depending on how rapidly the local potentials change. When the potential foliates into a continuum of onedimensional potentials, it is natural to use the transverse eigenstates provided by the transverse binding potentials. This becomes less intuitive when there are several degenerate minima, as the eigenstates in this case are delocalized having equal probability of being found in each. In these cases, although it has been shown that splitting is adequately described using the eigenstates [33], it seems natural to transform to a basis that allows for a description in terms of localized modes in the same way as is common in molecular physics using the method of hybridization to analyze bond localization [38]. The formalism described in Sec. III interpolates between a basis localized in a single minimum before the split and localization in each of the outgoing guides after the split. It should be observed that the equivalence between the upper (right) and lower (left) guides broken in an arbitrary way is correct, as the sign of the transformation angle given by Eq. (26), which can be chosen, is arbitrary but nevertheless gives a unique mapping between the transverse and localized states. The choice of mapping the ground state to either the upper or lower guide does not influence the dynamics, only the representation of it. We feel that the criterion used here is a natural choice if the new modes are to be localized in either well. The framework described in this work should find uses in simulations of interferometric devices as the manipulations lead to phase shifts between separated parts of matter wave packets, which act as independent states sensitive mainly to changes in the local binding potential valley. This makes the transverse eigenstates unsuitable as a basis since they are unable to properly take local perturbations into account when the guides are far separated. Obviously the localized states given by Eq. (28) offer a better choice. In this picture, the effective dynamics is formally identical to that of two weakly coupled single waveguides, each being sensitive only to local perturbations. For these states adiabatic propagation is limited by the same conditions as those that apply to single waveguides. In the present work we only outline the formalism and show the equivalence with adiabatic splitting in terms of transverse eigenstates.

Traditionally the conditions for validity of the adiabatic approximation are stated in terms of the time scales over which the Hamiltonian describing the system changes. In our earlier work we found connections between adiabaticity and the diffraction of matter waves. This made it possible to express these conditions in geometrical terms borrowed from classical optics. In Sec. III the regimes of validity of adiabatic propagation schemes based on transverse eigenstates versus ones based on localized states were outlined using heuristic arguments based on the relevant time scales. Connections were also made to our earlier work in order to relate the different regimes to the adiabaticity conditions given by intrinsic diffraction of matter waves. It would be desirable to rather state the validity criteria in geometrical terms in a more stringent way than has been done here. It appears likely that such an approach would yield further insights into the fundamental understanding of how matter waves are split in potential structures.

The behavior of coherent superpositions of transverse eigenstates in split potential structures is nontrivial. The reason is that it involves the relative phase between the propagating states, a quantity possibly varying with spatial coordinate. The resulting probability distribution after the split is conveniently described using the localized states instead of the transverse states which are delocalized and thus conceal information in the relative phases. A superposition wave packet of finite extent propagating through a potential split will be divided between the outgoing guides depending on its longitudinal extent relative to the distance propagated during one transverse oscillation. Observation of any asymmetry between the outgoing guides for wave packets prepared in superposition states can thus reveal information about the longitudinal extent of the state, something not possible in interferometric studies as there are alternative explanations equally valid [41].
[1] H. J. Metcalf, and P. van der Straten, Laser Cooling and Trapping (Springer, New York, 1999).

[2] V.I. Balykin, V.G. Minogin, and V.S. Letokhov, Rep. Prog. Phys. 63, 1429 (2000).

[3] W. Liang, M. Bockrath, D. Bozovic, J.H. Hafner, M. Tinkham, and H. Park, Nature (London) 411, 665 (2001); J. Li, D. Stein, C. McMullan, D. Branton, M.J. Aziz, and J.A. Golovchenko, ibid. 412, 166 (2001); M.S. Gudiksen, L.J. Lauhon, J. Wang, D.C. Smith, and C.M. Lieber, ibid. 415, 617 (2002); R. de Picciotto, H.L. Stormer, L.N. Pfeiffer, K.W. Baldwin, and K.W. West, ibid. 411, 51 (2001).

[4] C.S. Adams, M. Sigel, and J. Mlynek, Phys. Rep. 240, 143 (1994); J.P. dowling, and J. Gea-Banaloche, Adv. At., Mol., Opt. Phys. 37, 1 (1996); E.A. Hinds, and I.G. Hughes, J. Phys. D 32, R119 (1999).

[5] H.R. Noh, and W. Jhe, Phys. Rep. 372, 269 (2002).
[6] J. Reichel, Appl. Phys. B: Lasers Opt. 75, 469 (2002).

[7] T.J. Davis, J. Opt. B: Quantum Semiclassical Opt. 1, 408 (1999); M. Vengalattore, W. Rooijakkhers, and M. Prentiss, Phys. Rev. A 66, 053403 (2002); A.I. Sidorov, R.J. McLean, F. Scharnberg, D.S. Gough, T.J. Davis, B.J. Sexton, G.I. Opat, and P. Hannaford, Acta Phys. Pol. B 33, 2137 (2002).

[8] R. Grimm, M. Weidemüller, and Y.B. Ovchinnikov, Adv. At., Mol., Opt. Phys. 42, 95 (1999).

[9] G. Birkl, F.B.J. Buchkremer, R. Dumke, and W. Ertmer, Opt. Commun. 191, 67 (2001).

[10] D. Müller, E.A. Cornell, M. Prevedelli, P.D.D. Schwindt, A. Zozulya, and D.Z. Anderson, Opt. Lett. 25, 1392 (2000).

[11] D. Cassettari, B. Hessmo, R. Folman, T. Maier, and J. Schmiedmayer, Phys. Rev. Lett. 85, 5483 (2000).

[12] H. Ott, J. Fortágh, G. Schlotterbeck, A. Grossmann, and C. Zimmermann, Phys. Rev. Lett. 87, 230401 (2001). 
[13] W. Hänsel, P. Hommelhoff, T.W. Hänsch, and J. Reichel, Nature (London) 413, 498 (2001).

[14] S. Schneider, A. Kasper, C. vom Hagen, M. Bartenstein, B. Engeser, T. Schumm, I. Bar-Joseph, R. Folman, L. Feenstra, and J. Schmiedmayer, Phys. Rev. A 67, 023612 (2003).

[15] C.J. Vale, B.V. Hall, D.C. Lau, M.P.A. Jones, and E.A. Hinds, Europhys. News 33, 198 (2003).

[16] K. Bongs, S. Burger, S. Dettmer, D. Hellweg, J. Arlt, W. Ertmer, and K. Sengstock, Phys. Rev. A 63, 031602 (2000).

[17] A.E. Leanhardt, A.P. Chikkatur, D. Kielpinski, Y. Shin, T.L. Gustavson, W. Ketterle, and D.E. Pritchard, Phys. Rev. Lett. 89, 040401 (2002).

[18] R. Dumke, T. Müther, M. Volk, W. Ertmer, and G. Birkl, Phys. Rev. Lett. 89, 220402 (2002).

[19] J.F. Clauser, Physica B \& C 151, 262 (1988).

[20] M.O. Scully and J.P. Dowling, Phys. Rev. A 48, 3186 (1993).

[21] O. Zobay and B.M. Garraway, Opt. Commun. 178, 93 (2000).

[22] E.A. Hinds, C.J. Vale, and M.G. Boshier, Phys. Rev. Lett. 86, 1462 (2001).

[23] W. Hänsel, J. Reichel, P. Hommelhoff, and T.W. Hänsch, Phys. Rev. A 64, 063607 (2001).

[24] E. Andersson, T. Calarco, R. Folman, M. Andersson, B. Hessmo, and J. Schmiedmayer, Phys. Rev. Lett. 88, 100401 (2002).

[25] P. Meystre, AtomOptics (Springer, New York, 2000).

[26] M.D. Girardeau, Kunal K. Das, and E.M. Wright, Phys. Rev. A 66, 023604 (2002).

[27] J.A. Stickney and A.A. Zozulya, Phys. Rev. A 66, 053601 (2002).

[28] U. Dorner, P. Fedichev, D. Jaksch, M. Lewenstein, and P. Zoller, e-print quant-ph/0212039.
[29] M. A. Nielsen, and I. L. Chuang, Quantum Computation and Quantum Information (Cambridge University Press, Cambridge, 2000).

[30] T. Calarco, E.A. Hinds, D. Jaksch, J. Schmiedmayer, J.I. Cirac, and P. Zoller, Phys. Rev. A 61, 022304 (2000).

[31] R. E. Wyatt Dynamics of Molecules and Chemical Reactions, edited by J. Z. H. Zhang (Dekker, New York, 1996); N. Balakrishnan, C. Kalyanaraman, and N. Sathyamurthy, Phys. Rep. 280, 80 (1997); B.M. Garraway and K.A. Suominen, Rep. Prog. Phys. 58, 365 (1995).

[32] M. Jääskeläinen and S. Stenholm, Phys. Rev. A 66, 023608 (2002).

[33] M. Jääskeläinen (unpublished).

[34] M. Jääskeläinen and S. Stenholm, Phys. Rev. A 66, 043612 (2002).

[35] M. Jääskeläinen and S. Stenholm, Phys. Rev. A 66, 053605 (2002).

[36] A. W. Snyder and J. D. Love, Optical Waveguide Theory (Chapman and Hall, London, 1983).

[37] T. Dittrich, P. Hänggi, G.-L. Ingold, B. Kramer, G. Schön, and W. Zwerger, Quantum Transport and Dissipation (WileyVCH, Weinheim, 1998).

[38] R. L. Carter, Molecular Symmetry and Group Theory (Wiley, New York, 1998).

[39] H. Kaiser, S.A. Werner, and E.A. George, Phys. Rev. Lett. 50, 560 (1983).

[40] A.G. Klein, G.I. Opat, and W.A. Hamilton, Phys. Rev. Lett. 50, 563 (1983).

[41] G. Comsa, Phys. Rev. Lett. 51, 1105 (1983).

[42] A. Siegman, Lasers (University Science Books, Sausolito, 1986). 